\title{
Amide versus amine ratio in the discrimination layer of Reverse Osmosis membrane by solid state ${ }^{15} \mathrm{~N}$ NMR and DNP NMR
}

\section{Authors}

XiaoHua Qiu, David Redwine

Analytical Sciences, The Dow Chemical Company, Midland, Michigan 48667, U.S.A

Kebede Beshah

Analytical Sciences, The Dow Chemical Company, Collegeville, Pennsylvania 19426, U.S.A

Sara Livazovic

Middle East Innovation Center, The Dow Chemical Company, Thuwal, 23955-6900, Kingdom of Saudi Arabia

Christian G. Canlas, Andrei Guinov, Abdul-Hamid M. Emwas

Core Labs, King Abdullah University of Science and Technology, Thuwal, 23955-6900, Kingdom of Saudi Arabia 


\section{Abstract}

The state of the art membrane chemistry for reverse osmosis application is based on a classical interfacial polymerization reaction between a diamine in the aqueous phase and trifunctional acid chloride in the organic phase. Because of the very fast reaction rate of the interfacial polymerization, the extremely thin nature (typically $\leq 200 \mathrm{~nm}$ ), and the insolubility of the resulting polyamide layer, the conversion rate has not been directly studied. In this work, high field (21.2 Tesla) solid state NMR was utilized to directly measure amide to amine ratio of the polyamide layers in commercial RO membrane. Contrary to earlier indirect measurement, amines are rather abundant in these polyamide layers. Because of the dramatic reactant concentration differences on the opposing surfaces of the growing membrane, compositional heterogeneity is expected to exist across the membrane thickness. Dynamic nuclear polarization combined with solid-state NMR was utilized to probe the amide to amine ratio close to the membrane surface and was successful in differentiating membrane targeting different end user applications. The findings are important to understand both the interfacial polymerization chemistry as well as the performance of the resulting RO membrane since the amine groups present can form hydrogen bonds and ionize or deionize based on $\mathrm{pH}$. The surface amine groups can be further chemically modified to acquire additional properties for membranes.

\section{Introduction}

Water scarcity is one of the most serious global challenges facing the world today. [1] . Over one third of world's population lives in water-stressed countries with additional growth projected to nearly twothirds by year 2025. [2]. In many cases, natural water supplies are decreasing due to climate change and over exploration, and measures such as water conservation and water transport, or the construction of new dams are insufficient to cope with new demand. Developing desalination, a technology that purifies saline water into clean water, offers a practical solution to these problems. Reverse osmosis (RO) membrane technology is one of the most promising desalination technologies. Thin-film-composite (TFC) membranes for RO process are widely adopted in both residential water markets and many industrial areas such as desalination of brine [3], ultrapure water production, and etc. [4] Over the past three decades, aromatic polyamide membranes such as FT-30 of FilmTec Corp., which are produced through interfacial polymerization [5] of m-phenylenediamine (MPD) in the aqueous phase and trimesoyl chloride (TMC) in the organic phase have been established as the most commercially successful TFC RO membranes. This cross-linked, dense, but thin layer (200 nm) [6] of polyamide also known as the discrimination layer is formed on top of a microporous support (most often polysulfone) which is further supported by a polyester layer to provide sufficient mechanical strength. A representation of the different layers of RO membrane is shown in Figure 1. Commercial membranes typically have a humectant layer on top of the membrane that can be several microns thick. Some membranes can have coating layers in between the polyamide layer and the humectant layer. 


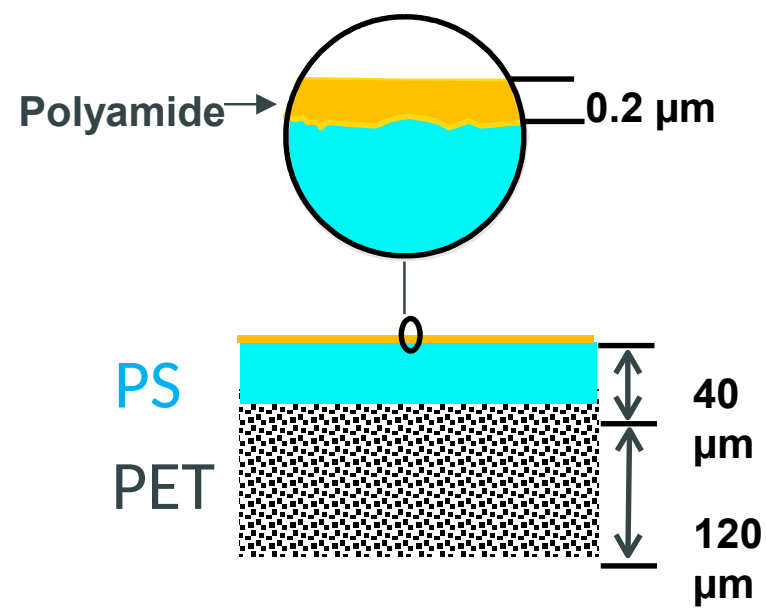

Figure 1. Representation of the different layers that make up reverse osmosis membranes.

The composition and the micro-structure of the polyamide discrimination layer which is critical to membrane performances are very difficult to elucidate because of the insoluble nature of the polymer and severe matrix interferences from both the supporting layer under it and coating layer above it. A general molecular structure is shown in Scheme 1. However the amount of amine and carboxylic acid group relative to amide group is not known. The most applied technologies to study this polyamide layer are methods developed to probe surfaces including scanning electron microscopy [7], transmission electron microscopy [8], and atomic force microscopy [9] that universally show a typical rough pattern. Streaming potential measurements along the membrane surface indicated that the membrane possesses negative charge. [10] X-ray photoelectron spectroscopy(XPS) and FTIR (after correction for the polysulfone spectral feature) were also employed to study the surface and bulk of the membrane respectively [11] and solid state NMR were utilized to analyze the dynamics of thick free standing polyamide layer [12]. Positron Annihilation Spectroscopy was applied to measure the free volume in the layer [13]. The most promising technology to study the functional groups in the polyamide layer is Rutherford backscattering spectroscopy (RBS) [14]. Deprotonated carboxylic groups were tagged with $\mathrm{Ag}^{+}$, and protonated amine groups with $\mathrm{WO}^{2-}$ in order for these functional groups to be monitored by RBS. 


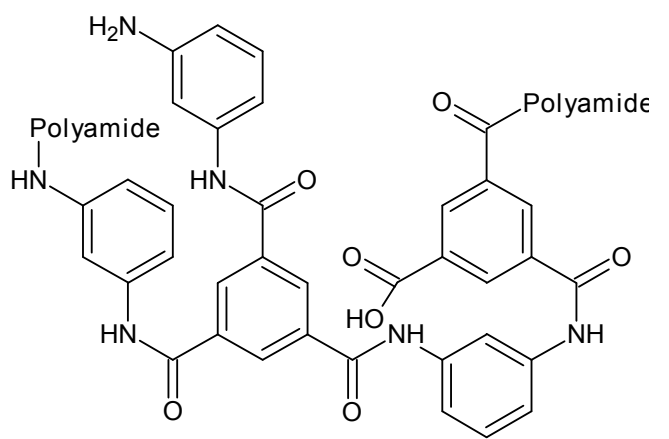

Scheme 1. General structure of polyamide membrane

Both XPS and RBS can provide information about the elemental composition of the polyamide layer. Kim et al. [13] measured C:N:O ratio of 73.7:10.9:15.4 with a N/O ratio of 0.71 using XPS on FT30 membrane. Coronell et al. 2008 measured $\mathrm{C}: \mathrm{H}: \mathrm{N}: \mathrm{O}$ ratio of 48:33:7:12 (C:N:O ratio of 71.6:10.4:17.9) with a N/O ratio of 0.58 using RBS on FT30 membrane as well as carboxylic acid and amine function groups at 0.423 and $0.036 \mathrm{M}$. Both methods indicate less $\mathrm{N}$ than $\mathrm{O}$ in the layer which implies a relatively sparse amine concentration. These methods can be complicated by various humectants and coating layers manufacturers put on membranes post interfacial polymerization. Since these additives are typically deficient in $\mathrm{N}$ and rich in $\mathrm{C}$ and $\mathrm{O}$, the interpretation of the composition from elemental analysis data can be ambiguous.

In this study, direct measurement of nitrogen chemical environment (amide versus amine) was accomplished by solid state ${ }^{15} \mathrm{~N}$ Nuclear Magnetic Resonance (NMR) spectroscopy. Since the polyamide layer is usually the only source of $\mathrm{N}$ in $\mathrm{RO}$ membrane, this technique removes all the ambiguities about signal contamination from coating and supporting layer. Ultra-high magnetic field at 21.1 tesla (900 $\mathrm{MHz}$ for ${ }^{1} \mathrm{H}$ ) and polyamide isolation were used to overcome the low sensitivity problem of ${ }^{15} \mathrm{~N} \mathrm{NMR}$. Dynamic Nuclear Polarization (DNP) NMR was applied to investigate the nitrogen chemical environment close to the surface.

Amine functional groups are believed to play an important role in membrane performance since they can form hydrogen bonds and can be ionized and deionized depending on $\mathrm{pH}$. The ratio of amine to amide also provides information about the conversion rate for the interfacial polymerization. Primary amine can be reactive and provides an anchor for further membrane modification. [15] Therefore, a method to probe amine functional groups is an indispensable tool to understand membrane chemistry and future membrane development. Carboxylic acid groups can also play an important role similar as amine groups. They are not the focus of this study because of other carbon and oxygen containing species in a typical membrane environment. In general the less complete the conversion rate as measured by the amine to amide ratio, the more carboxylic acid groups are available in the membrane as well. 


\section{Experimental}

\section{Reagents and Materials}

$\mathrm{SWXHR}{ }^{\mathrm{TM}}, \mathrm{BW} 30^{\mathrm{TM}}$, and XLE ${ }^{\mathrm{TM}}$ RO membranes were provided by the FilmTec Corporation. Polyamide layers were isolated using the following procedure: First, the polyester backing was physically peeled off by initiating a small tear then while holding the polysulfone side securely down against a flat surface, slowly pull the polyester away from the membrane. With practice, the polysulfone layer and all the layers above it can be peeled off in a single piece. The polysulfone layer were then washed with copious amounts of N,N-dimethyl formamide (DMF) four times to dissolve the polysulfone. The remnant was then washed thrice with water to remove DMF and thrice with methanol to remove water. The dried membrane was further dried in a vacuum oven at $50{ }^{\circ} \mathrm{C}$ overnight to remove methanol. Solvent extraction of this delaminated membrane did not show any detectable DMF, methanol, or MPD at ppm level.

Small polyamide fragments not cross-linked to the bulk discrimination layer may be lost during the washing process. The yield of each washing process was carefully monitored and matched the expectation of the area sampled and the expected thickness of the discrimination layer. Other solvents, such as methylene chloride were tried and similar yields were generated in those trials. Thus no evidence of significant discrimination layer loss can be found in the preferred washing process. DMF was selected as the solvent of choice because of its low vapor pressure and thus low toxicity risk to the operator.

Nylon-6, polyaniline, MPD were acquired from Aldrich and used as is. 4-aminobenzanilide and N,N'-1,4phenylenebis(3-aminobenzamide) were acquired from Hit2Lead and assayed (95.1\% and 93.1\% pure respectively) using solution ${ }^{1} \mathrm{H}$ NMR. Nylon- 6 and polyaniline were selected as substitutes for immobile amide and aniline since they are well characterized and easily handled for solid state NMR sample preparation.

\section{Measurement by solid-state NMR}

For high field solid state NMR, approximately $10 \mathrm{mg}$ of delaminated membrane were put in inserts with screw-in cap appropriate for $4 \mathrm{~mm}$ O.D. zirconia rotors, tightly close, and put in the rotors. NMR spectra were obtained using a $4 \mathrm{~mm}$ double resonance low-gamma CP/MAS probe in a $900 \mathrm{MHz}$ Bruker Avance III WB (21.2 Tesla) instrument. The rotor spinning speed was set at $13 \mathrm{kHz}$. Typical acquisition parameters are: $90^{\circ}$ pulse width of $4 \mu \mathrm{s}$; cross polarization time of $2-4 \mathrm{~ms}$; acquisition time of $23 \mathrm{~ms}$; relaxation delay of 10 seconds; spectral width is set at $735 \mathrm{ppm}$ with the center of the spectrum set at 90 ppm. Approximately 40,000 scans were collected for data averaging. The chemical shift of the spectrum was referenced using an external standard of glycine at $33.4 \mathrm{ppm}$.

\section{Measurement by DNP solid-state NMR}

For DNP NMR, an amount of delaminated sample to fill the rotor was put in $3.2 \mathrm{~mm}$ O.D. sapphire rotors, weighed, and $40 \mu$ of DNP-radical containing solvent was added using the centrifuge. Rotor was sealed with a plug and a cap. The spectra were taken in a $400 \mathrm{MHz}$ Bruker Avance III HD WB DNP (9.4 
Tesla/263 GHz) instrument equipped with a Bruker $3.2 \mathrm{~mm}$ triple resonance ${ }^{1} \mathrm{H} /{ }^{13} \mathrm{C} /{ }^{15} \mathrm{~N}$ Low Temperature MAS probe spinning at 8-10 kHZ and temperature kept at $100 \mathrm{~K}$. Five DNP-radical containing solvent were employed:

1) 20 mM Amupol in Glycerol- ${ }^{12} \mathrm{C}_{3}, \mathrm{D}_{8} 60 \% ; \mathrm{D}_{2} \mathrm{O} 30 \%, \mathrm{H}_{2} \mathrm{O} 10 \%$;

2) $20 \mathrm{mM}$ amupol in Glycerol- ${ }^{12} \mathrm{C}_{3}, \mathrm{D}_{8} 60 \% ; \mathrm{H}_{2} \mathrm{O} 40 \%$;

3) $16 \mathrm{mM}$ TekPol in TCE;

4) $12 \mathrm{mM}$ Amupol in Glycerol 60\%; $\mathrm{H}_{2} \mathrm{O} 40 \%$;

5) $12 \mathrm{mM}$ Amupol in DMSO- $\mathrm{d}_{6} 60 \%$; $\mathrm{H}_{2} \mathrm{O} 40 \%$;

The $5^{\text {th }}$ one was found to provide best enhancement and employed for all the data presented in this manuscript. Typical acquisition parameters are: $90^{\circ}$ pulse width of $4 \mu \mathrm{s}$; cross polarization time of $2-4$ ms; acquisition time of $32 \mathrm{~ms}$; relaxation delay of 10 seconds; spectral width is set at $400 \mathrm{ppm}$ with the center of the spectrum set at $50 \mathrm{ppm}$. Approximately 128 scans were collected for data averaging and 4000 scans were collected when microwave is turned off to measure bulk composition.

\section{Results and Discussion:}

I. Qualitative High field Solid State NMR of Polyamide Discrimination Layer 


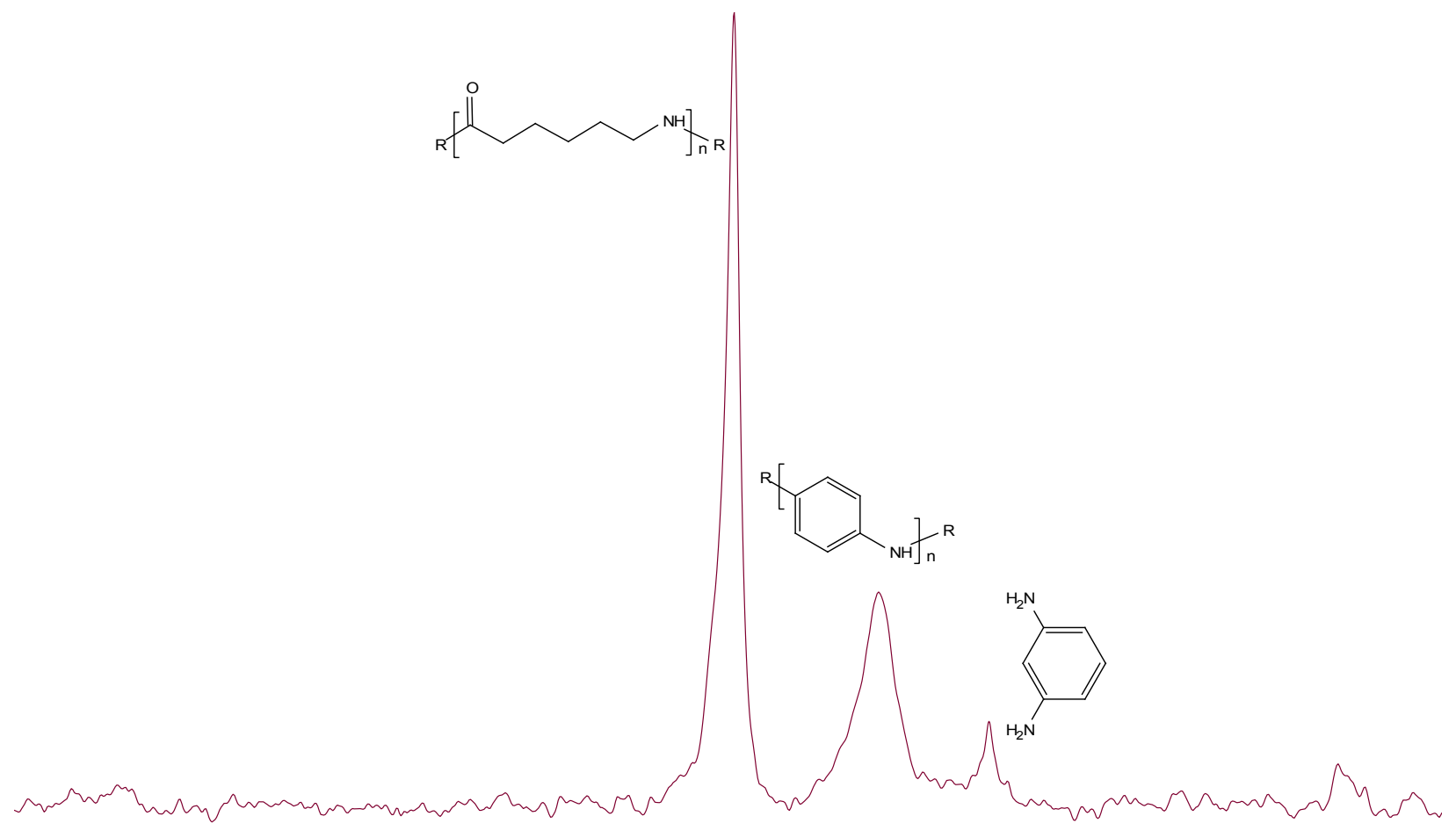

$\begin{array}{llllllllllllllllllllllllllllllllllllllllllllllllll}280 & 270 & 260 & 250 & 240 & 230 & 220 & 210 & 200 & 190 & 180 & 170 & 160 & 150 & 140 & 130 & 120 & 110 & 100 & 90 & 80 & 70 & 60 & 50 & 40 & 30 & 20 & 10 & 0 & -10 & -20 & -30 & -40 & -!\end{array}$

Figure 2. ${ }^{15} \mathrm{~N} \mathrm{CP}(4 \mathrm{~ms}) / \mathrm{MAS}(13 \mathrm{KHz})$ spectrum of an equimolar blend of Nylon-6 (118 ppm), polyaniline (82 ppm), and MPD (57 ppm). The peaks at 262 and -25 ppm are spinning side bands.

Figure 2 showed an ${ }^{15} \mathrm{~N}$ solid state CP/MAS spectrum of a physical blend of equimolar Nylon-6, polyaniline, and MPD demonstrating all three nitrogen chemical environment can be clearly resolved. Figure 3 showed a multiCP/MAS spectrum of the delaminated polyamide layer. It is clear that both amide and amine functional groups can be clearly identified and amine concentration is substantial. To our knowledge, this is the first time that amine functionality has been directly observed in the polyamide discrimination layer of RO membrane. Over 16000 scans were collected for data averaging which makes this experiment exotic rather than routine analysis. 


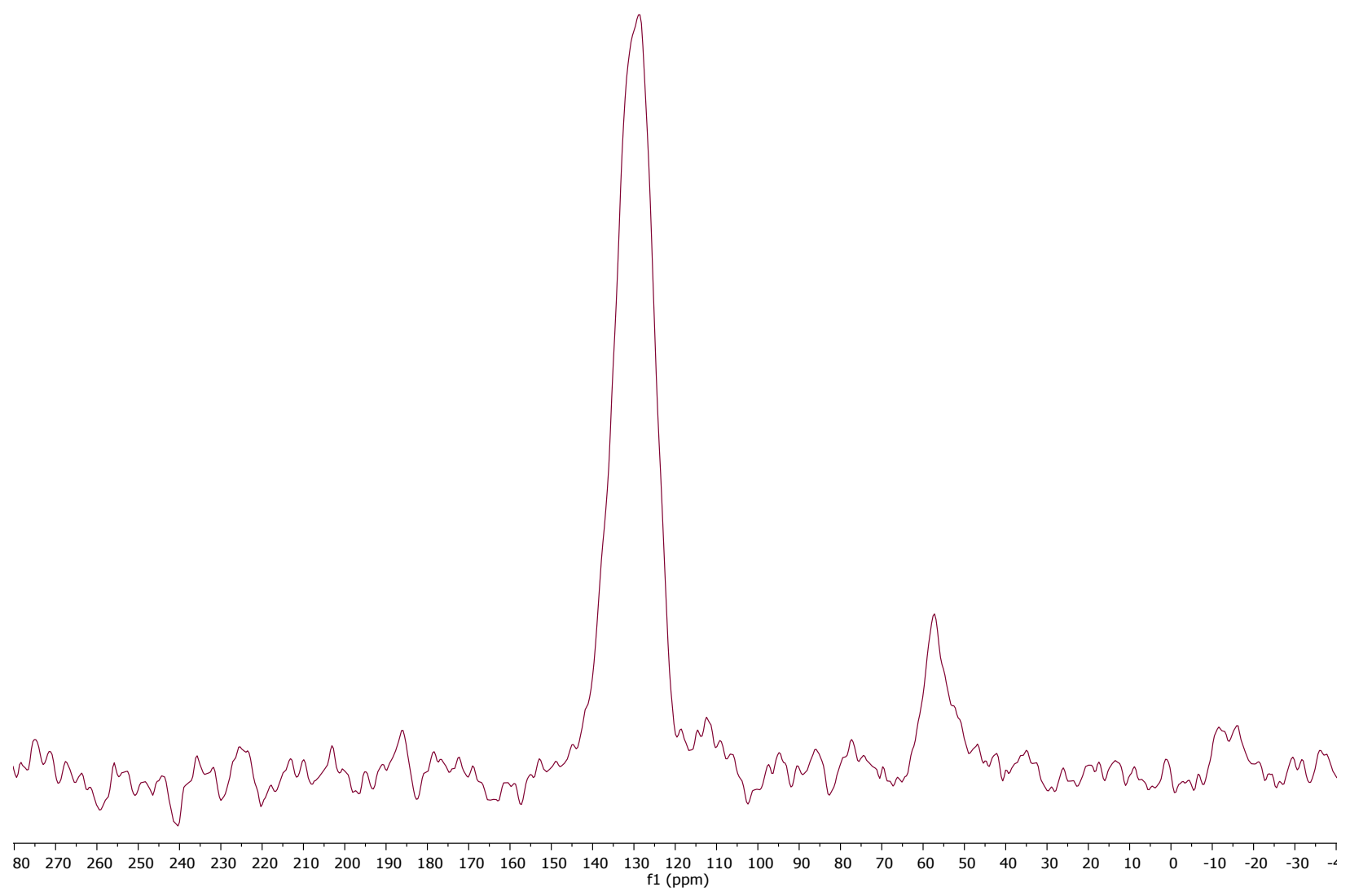

Figure 3. ${ }^{15} \mathrm{~N}$ multiCP/MAS spectrum of delaminated polyamide layer of BW30 RO membrane.

II. Qualitative Solid State DNP NMR of Polyamide Discrimination Layer

Figure 4 showed the comparison of microwave off and microwave on DNP/CP/MAS ${ }^{15} \mathrm{~N}$ spectra of delaminated BW30 membrane. With optimal radical/solvent pair and acquisition parameters, enhancement of 20 can be achieved which brings the experiment time down to a practical level. For the microwave on experiment, not only the experimental time is less than one quarter of the time than the one shown in Figure 3, but the signal to noise ratio improved significantly as well. This improvement makes such experiments practical. Qualitatively, the main amide peak with a substantial amine peak can still be observed showing there is no dramatic changes in composition from the surface specific enhancement of DNP. 

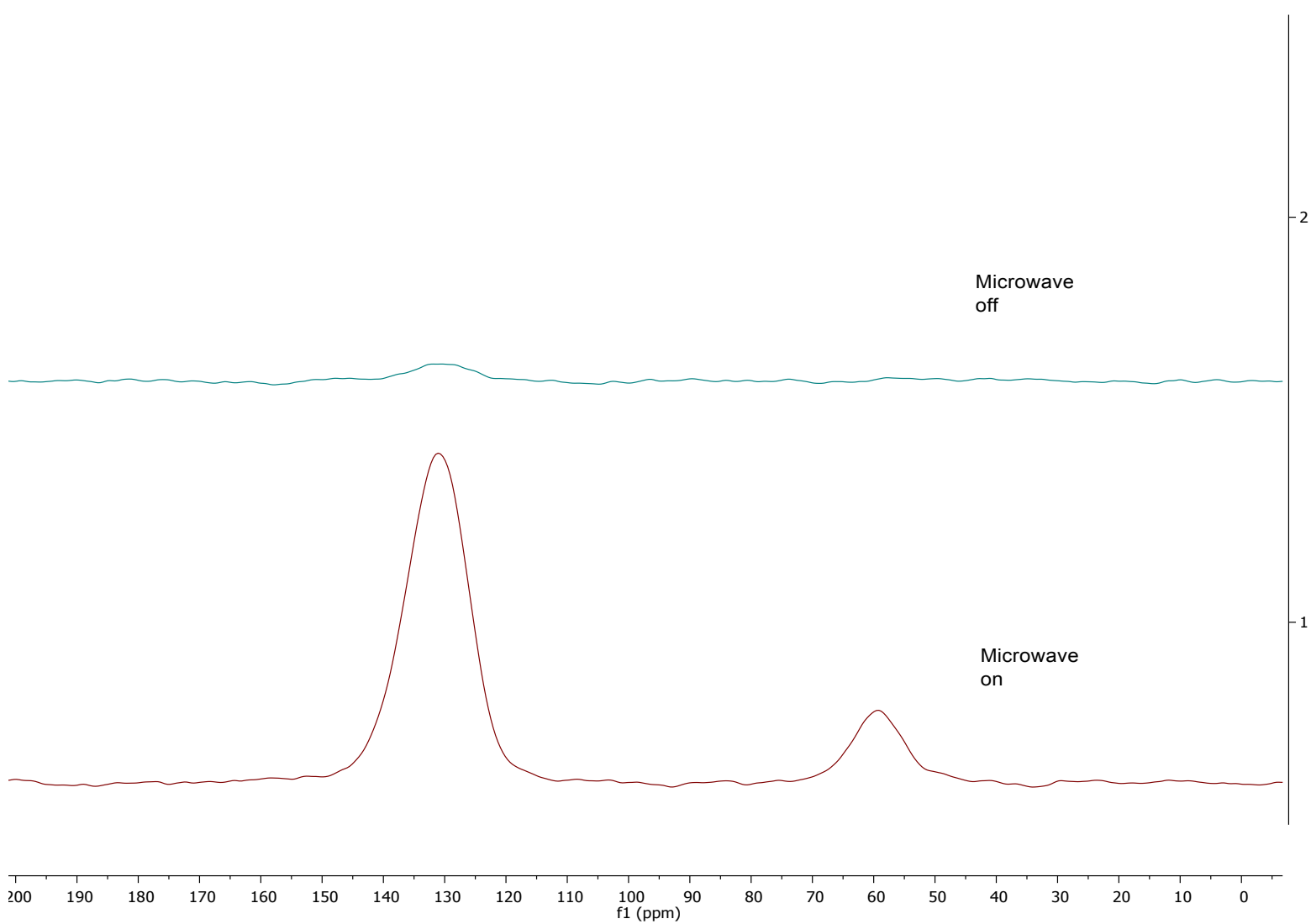

Figure 4. ${ }^{15} \mathrm{~N}$ DNP/CP/MAS spectrum of delaminated polyamide layer of BW30 RO membrane. Top spectrum is microwave off and bottom spectrum is microwave on.

Spectrum was also collected for non-delaminated (only the PET layer was removed physically by peeling) RO membrane (shown in Figure 5). Considering only $0.5 \%$ of the sample (the polysulfone layer is around 50 micron thick and the polyamide is around $200 \mathrm{~nm}$ thick) is polyamide, the detection of amide signal at $135 \mathrm{ppm}$ is a rather amazing testament to the signal enhancement capability of DNP NMR (at $80,000+$ scans or $222+\mathrm{hrs}$ ). In addition to the amide peak, polyaniline type peak was also observed around $80 \mathrm{ppm}$. They are likely from residual MPD in the membrane that self oligomerized/polymerized overtime. Possible locations for these remnants of residual MPD can be in polyamide layer or polysulfone layer or both. Since aged membrane usually has a brown tint on the polyamide surface but completely white on the polysulfone surface, the polyaniline signal is hypothesized to come from the polyamide surface. Qualitatively this signal is about the same magnitude as the amide signal. This observation should suggest caution in any polyamide composition analysis based on non-delaminated membrane since substantial amount of residual MPD exist and its chemical structure can change over time with oligomerization reaction. It also demonstrated that solid state NMR can easily distinguish nascent amine from its oxidized version through chemical shift differences. Although DNP is a very powerful technique to probe polyamide surface functional groups, the rest of the manuscript concentrates on delaminated membrane to study the delaminated polyamide layer composition. 


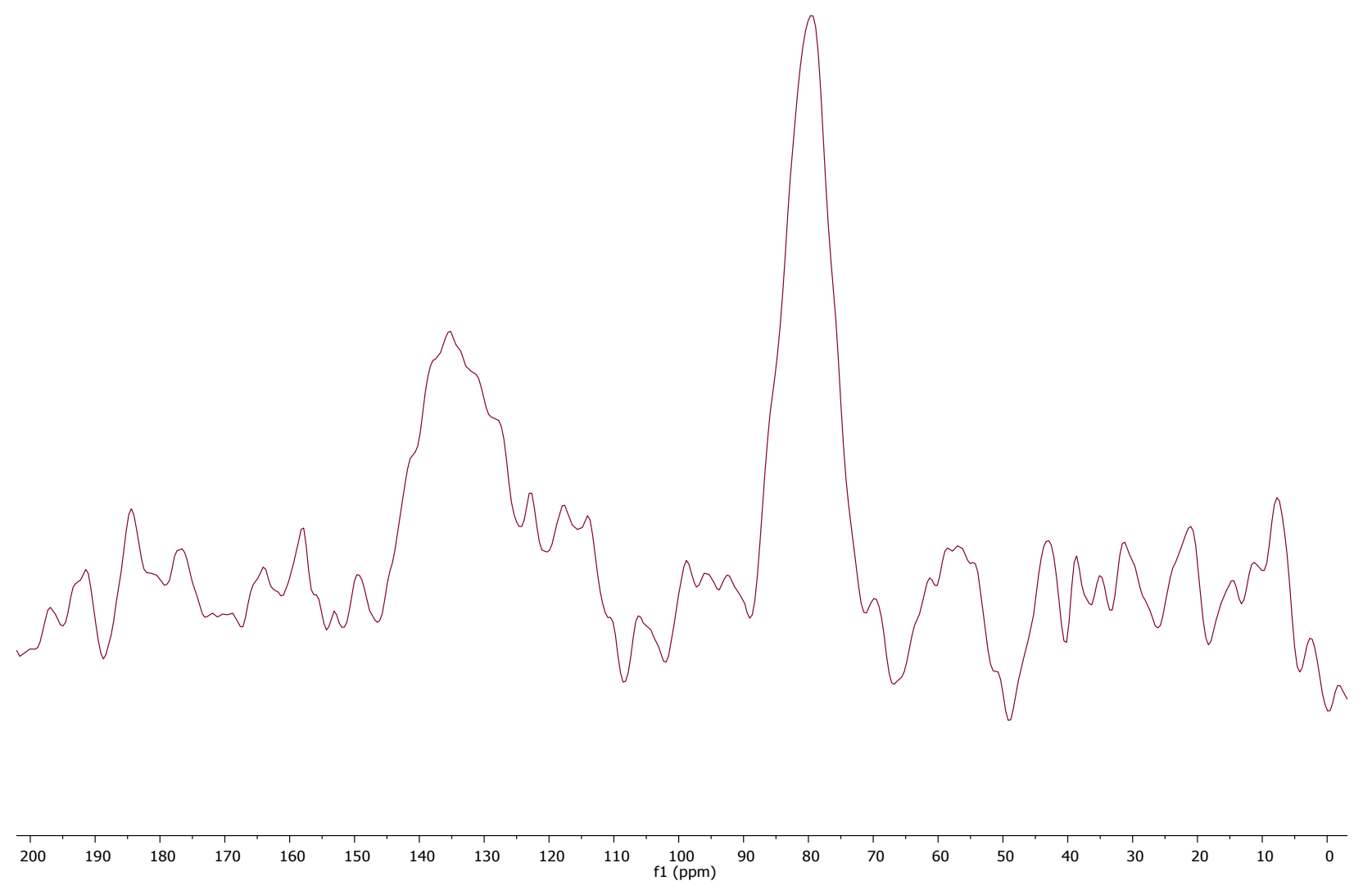

Figure $5 .{ }^{15} \mathrm{~N} D N P / C P / M A S$ spectrum of the non-delaminated polyamide layer of BW30 RO membrane.

III. Quantitative High field Solid State NMR of Polyamide Discrimination Layer

In Figure 2, the relative intensity for MPD is only a fraction of the other two even though it is an equimolar blend. MPD signal also decreased significantly with increasing CP time but nylon-6 and polyaniline did not show significant signal decrease with increasing CP time. In fact, if not mixed with other large molecules, MPD alone did not show any signal beyond limit of detection with CP experiments. Only in the high power decoupling mode can signal from MPD alone sample be detected. All of the observations about MPD suggested the mobility of MPD is a problem to quantitate such species in CP experiment. 

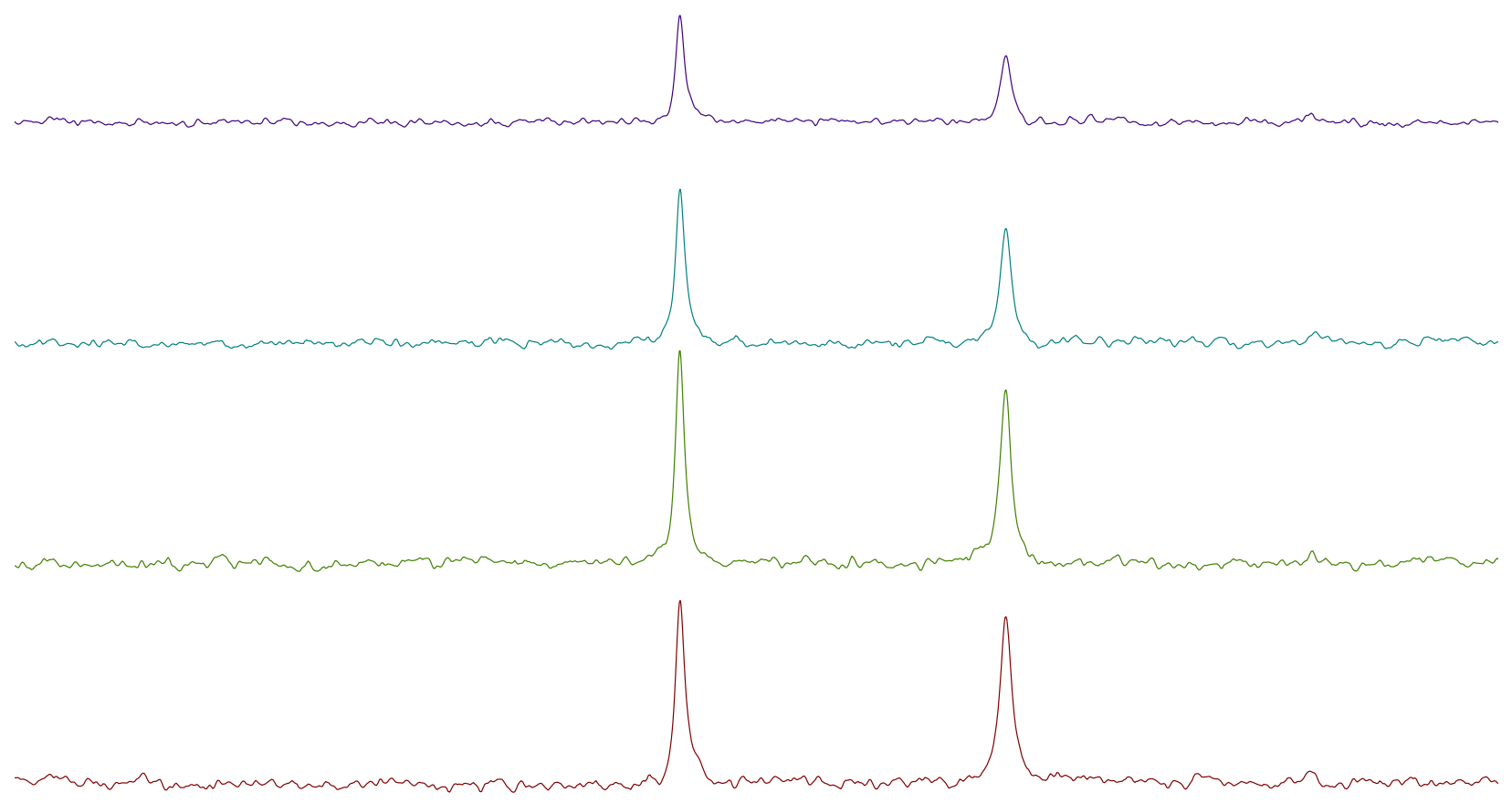

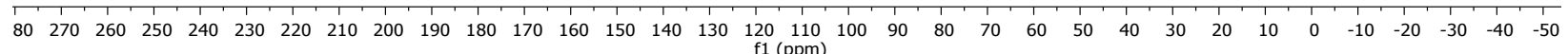

Figure $6 .{ }^{15} \mathrm{~N}$ CP/MAS spectrum of N,N'-1,4-phenylenebis(3-aminobenzamide) with CP time of $0.5 \mathrm{~ms}$ (bottom), $1 \mathrm{~ms}$ (second from bottom), $2 \mathrm{~ms}$ (second from top), and $3 \mathrm{~ms}$ (top).

Figure 6 shows solid state ${ }^{15} \mathrm{~N}$ CP/MAS NMR of N,N'-1,4-phenylenebis(3-aminobenzamide) (structure shown in Scheme 2) with various $\mathrm{CP}$ time. This molecule has two amides and two amines. It is clear from the plot that the relative intensity of amine to amide decreases with increasing CP time, with amine signal only $80 \%$ of the amide signal at $2 \mathrm{~ms}$ CP time. Similar experiments on 4-aminobenzanilide, another molecule with one amine and one amide (shown in Supplemental Material) shows the amine signal is only $50 \%$ of the amide signal at $2 \mathrm{~ms}$ CP time. 


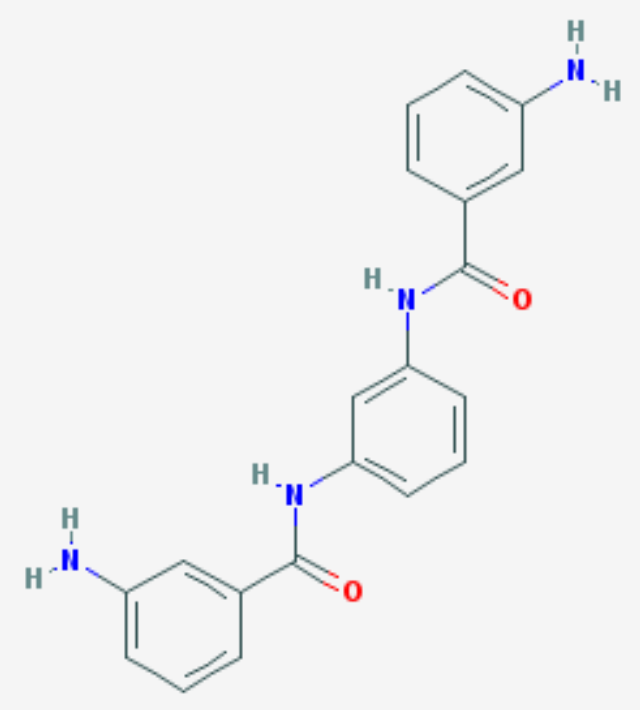

Scheme 2. Chemical structure of N,N'-1,4-phenylenebis(3-aminobenzamide)

Given the observation that MPD (MW $108 \mathrm{~g} / \mathrm{mol}$ ) has no CP signal, and only has CP signal when mixed with larger polymers such as nylon or polyaniline and signal decreases with increasing CP time; 4aminobenzanilide (MW $212 \mathrm{~g} / \mathrm{mol}$ ) has amine to amide integral ratio of 1:2 at 2 ms CP time; and N,N'1,4-phenylenebis(3-aminobenzamide) ( $\mathrm{MW} 346 \mathrm{~g} / \mathrm{mol}$ ) has nominal amine to amide integral ratio decreasing with increasing CP time, the exact integral ratio at $2 \mathrm{~ms} C P$ time is 1:1.2, we hypothesize that the sensitivities of amine signal to CP time is an indication of the mobility of the functional group. The molecular weight of polyamide discrimination layer is not known but should be significantly higher than the three standards listed in the previous paragraph. Therefore, the relative magnetization decay of amine with increasing CP time for the amine group should be less severe and has ratio closer to unity at $2 \mathrm{~ms} \mathrm{CP}$ experiment than a ratio of 1:1.2 for amine to amide ratio for a $\mathrm{MW}=346 \mathrm{~g} / \mathrm{mol}$ standard. Error bar estimation is $+/-20 \%$ if $\mathrm{S} / \mathrm{N}$ ratio of the peak is greater than 30 . However for room temperature 900 $\mathrm{MHz}$ data, the amine signal typically has a SNR of around 5 so the error bar estimation are $+/-50 \%$ relative for these samples.

Therefore the amine to amide ratio in the spectrum in Figure 3 is $0.14(+/-0.07): 1$, indicating approximately one in five MPD repeat units was not fully converted into diamide but rather one amide and one amine. This result is not consistent with the RBS results measuring more than 10 times carboxylic acid group as amine group. The result is also not consistent of the atomic ratio of $\mathrm{N}$ to $\mathrm{O}$ of 0.7 as suggested by both XPS and RBS experiments. Both of these experiments suffer from the potential contamination effects of coatings on membrane surface. The results validated our hypothesis that ${ }^{15} \mathrm{~N}$ solid state NMR offered a straight forward way to measure nitrogen containing species in the polyamide layer. 
Three commercial RO membranes(SWXHR ${ }^{\mathrm{TM}}, \mathrm{BW} 30^{\mathrm{TM}}$, and $\left.\mathrm{XLE}^{\mathrm{TM}}\right)$ were investigated using room temperature $900 \mathrm{MHz}{ }^{15} \mathrm{~N} \mathrm{CP} / \mathrm{MAS} N \mathrm{NR}$ and shown in Figure 7. All of them have detectable amine signal in addition to amide signal. Within the rather poor $\mathrm{S} / \mathrm{N}$ ratio of the spectrum (each with 4096 scans), there are no experimentally significant differences in the three membranes.

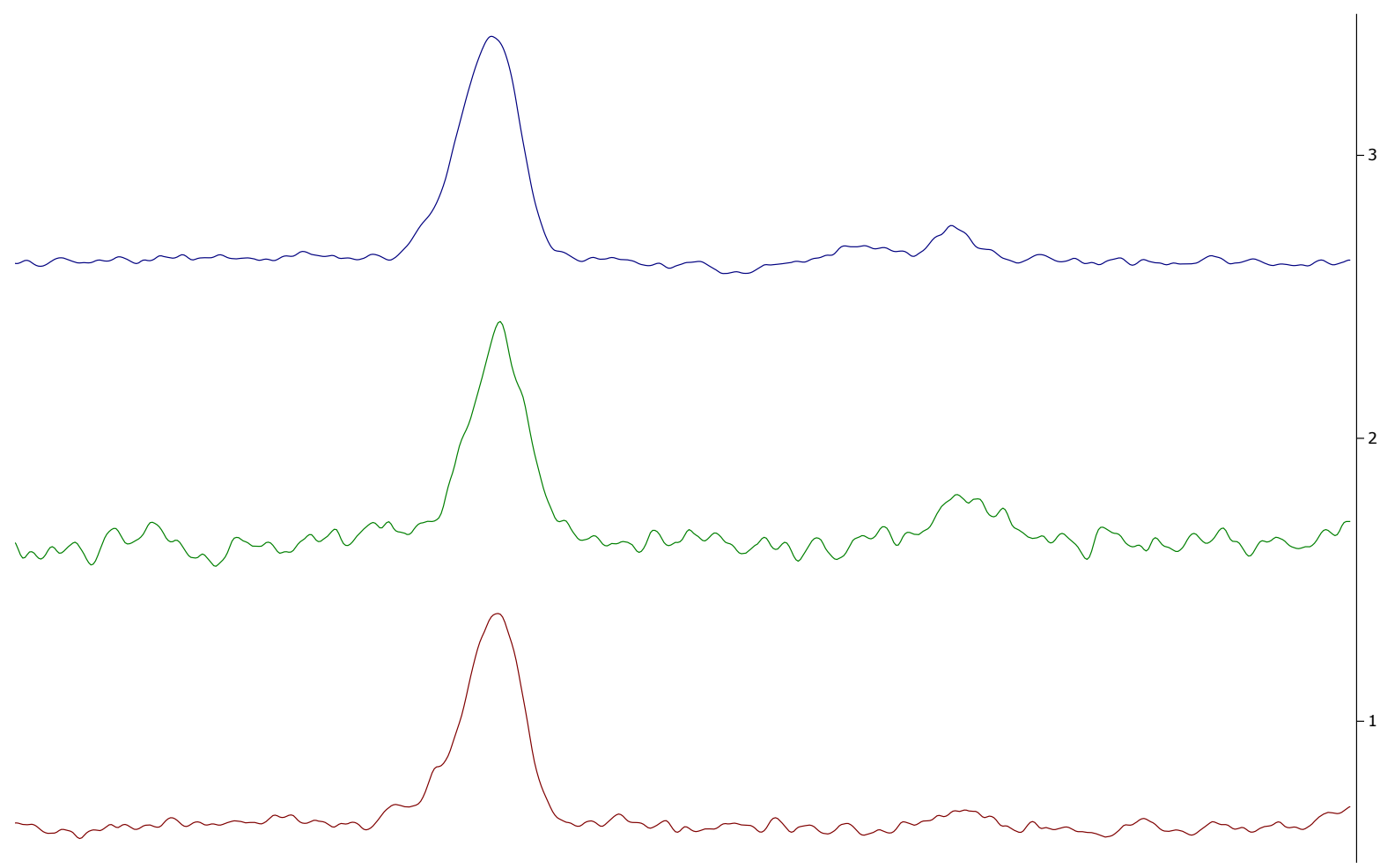

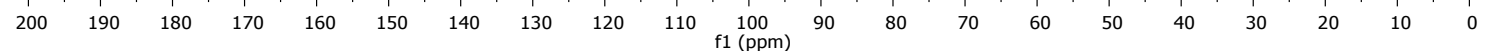

Figure $7 .{ }^{15} \mathrm{~N} C P / M A S$ spectrum of delaminated SWXLE (top), BW30 (middle), BWXLE (bottom).

IV. Quantitative DNP Solid State NMR of Polyamide Discrimination Layer

DNP NMR was run at $100 \mathrm{~K}$ using a $400 \mathrm{MHz}$ magnet and the sample is prepared with biradical glass forming solution. Figure 8 shows the comparison of a spectrum taken at that condition but with microwave off superimposed on a spectrum taken with room temperature $900 \mathrm{MHz}$ setup. Both spectra detect bulk without emphasis of surface. Other than minor broadening that can be attributed from the field difference and maybe biradical presence, the two spectra are very similar showing the cooling and biradical presence did not change the relative CP efficiency of amine vs. amide. 


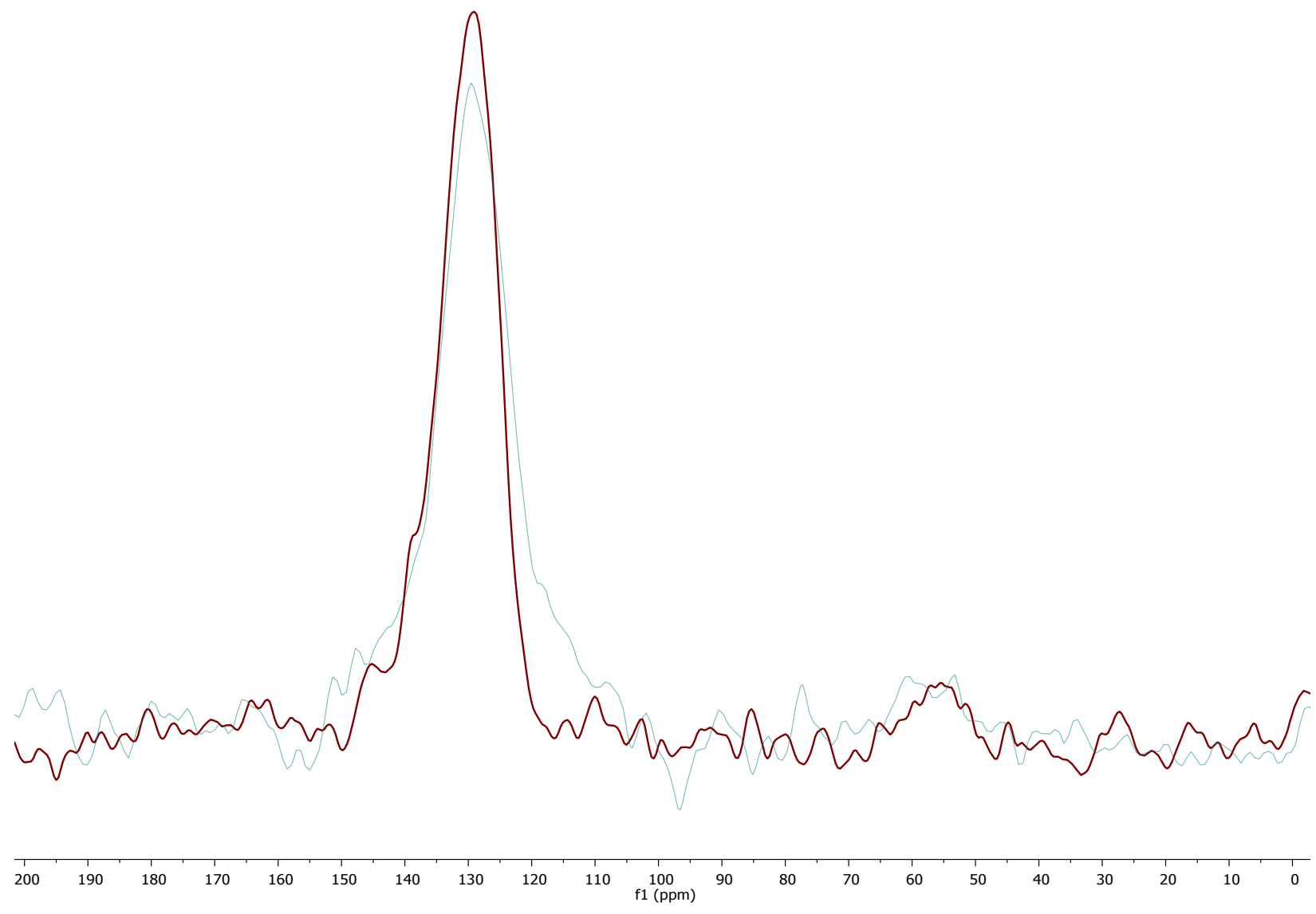

Figure $8 .{ }^{15} \mathrm{~N} \mathrm{CP/MAS} \mathrm{spectra} \mathrm{of} \mathrm{delaminated} \mathrm{XLE} \mathrm{membrane.} \mathrm{The} \mathrm{red} \mathrm{trace} \mathrm{is} \mathrm{at} \mathrm{room} \mathrm{temperature} 900 \mathrm{MHz}$ and the blue trace is the DNP prepared sample at $100 \mathrm{~K} 400 \mathrm{MHz}$ but with microwave off.

When microwave is turned on, signal is greatly enhanced through DNP effect and error bar associated with low signal to noise ratio can be greatly improved. However, the polyamide sample is extensively crosslinked and not permeable to any molecule significantly larger than $\mathrm{H}_{2} \mathrm{O}$ molecule by design, so the biradical distribution is not even throughout the sample. The surface signal is enhanced and the bulk signal is less affected by the DNP effect. The polyamide layer has asymmetrical surfaces because it is manufactured through interfacial polymerization where the top surface is exposed to oil phase that is rich in trimesoyl chloride and the bottom (support layer facing) layer is coated with aqueous solution of MPD. During delamination, it is observed that membrane curl up first and slowly lose its supporting layer of polysulfone. It is hypothesized that the majority of exposed surface for DNP NMR sample is also the top layer. 


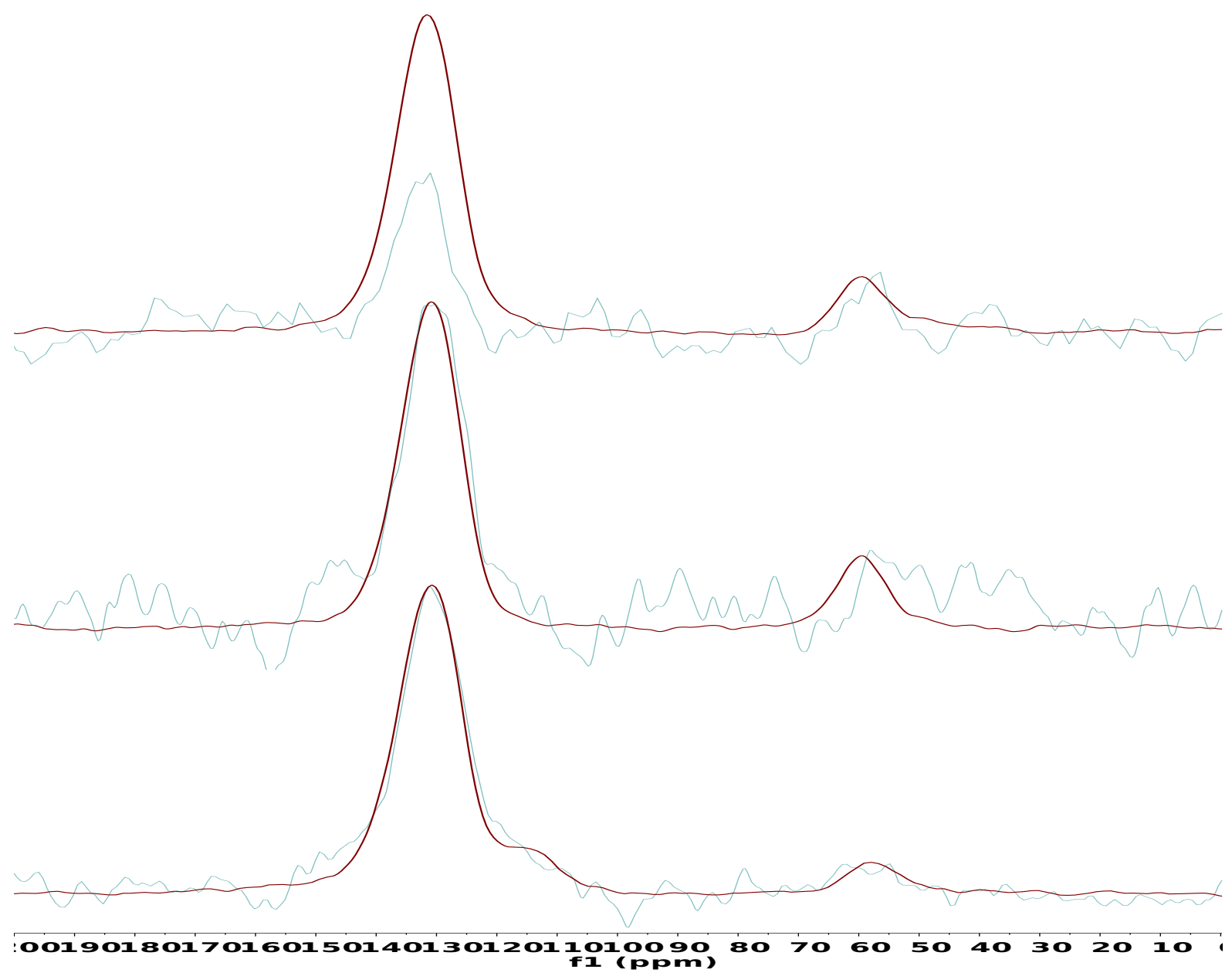

Figure 9. ${ }^{15} \mathrm{DNP} / \mathrm{CP} / \mathrm{MAS}$ spectra of delaminated SWXHR (top), BW30 (middle), and XLE (bottom) membrane. The blue traces are microwave off and red traces are microwave on.

Figure 9 showed the DNP enhanced CP/MAS spectra of delaminated SWXHR ${ }^{\mathrm{TM}}$, BW30 ${ }^{\mathrm{TM}}$, and $\mathrm{XLE}^{\mathrm{TM}}$ membrane. Both microwave off and microwave on spectra were shown in the Figure providing information for bulk and surface respectively. SWXHR ${ }^{\mathrm{TM}}$ showed more enhancement for the amide signal than amine signal indicating the surface layer is deficient in amine compared to the bulk. For $\mathrm{BW} 30^{\mathrm{TM}}$, the signal with microwave off is too poor to be certain. For $\mathrm{XLE}^{\mathrm{TM}}$, the enhancement factor for amine and amide are similar. There is a shoulder at $\sim 115 \mathrm{ppm}$ that was not present in the other samples and maybe contributed to nitrogen contaminations in the coating material that survived the delamination process. Since this contamination is not observed in the bulk spectrum it is likely a minor component but get preferentially enhanced by DNP. XLE ${ }^{\mathrm{TM}}$ also features substantially smaller amine to amide ratio in its DNP spectrum than the spectra for $\mathrm{SWXHR}{ }^{\mathrm{TM}}$ and $\mathrm{BW} 30^{\mathrm{TM}}$, indicating a more completely converted crosslinking system at least for the surface layer. Compared to room temperature $900 \mathrm{MHz}$ data, DNP NMR has greater signal to noise ratio and allowed us to differentiate among the membranes. 


\section{Conclusion}

${ }^{15} \mathrm{~N}$ solid state NMR is demonstrated to be a great tool to study the composition of polyamide discrimination layer on RO membrane. Room temperature $900 \mathrm{MHz}$ data directly observed substantial amount of amine, on the order of $1 / 10^{\text {th }}$ of amides. DNP NMR enhanced the signal sufficiently to make this experiment practical and were able to differentiate RO membranes targeted for different market segments. Polyaniline type of oligomerized/polymerized MPD were identified on the surface of nondelaminated membrane. Now that DNP NMR of RO membrane is demonstrated, many other established NMR structure and dynamics methods can be applied to further elucidate the structureproperty correlation for RO membranes. The amine to amide ratio is only a piece of puzzle for the interfacial polymerization and the resulting cross-linked polyamide. Further elucidating methods need to be developed on carboxylic acid to amide ratio to complete this puzzle to make structure-property relationship for the discrimination layer of RO membranes.

\section{Acknowledgement}

The authors would like to thank funding from the Dow Chemical Company and KAUST for this work. Supports from Dr. Paul O'Connor, Dr. Kun Li, Dr. Phil Griggs, and Dr. Steven Guillaudeu are essential for the collaboration between the two entities and this work.

\section{References}

[1] M. Elimelech and W. Phillip, "The Future of Seawater Desalination: Energy, Technology and the Environment," Science, p. 712, 2011.

[2] P. Raskin, P. Gleick, G. Pontius and K. Strzepek, "Water Futures: Assessment of Long-Range Patterns and Problems. Comprehensive Assessment of the Freshwater Resources of the World," Stockholm Environment Institute, Stockholm, 1997.

[3] J. Cadotte, R. Petersen, R. Larson and E. Erickson, "A new thin-film composite seawater reverse osmosis membrane," Desalination, pp. 25-31, 1981.

[4] M. Muler, Basic Principles of membrane Technology, Boston, MA: Kluwer Academic, 1996. 
[5] J. E. Cadotte.U.S.A Patent US4277344 A, 1981.

[6] V. Freger, "Nanoscale Heterogeneity of Polyamide Membranes Formed by Interfacial Polymerization," Langmuir, pp. 4791-4797, 2003.

[7] S.-Y. Kwak, S. Jung, Y. Yoon and D. Ihm, "Details of surface features in aromatic polyamide reverse osmosis membranes characterized by scanning electron and atomic force microscopy," Journal of Polymer Science Part B: Polymer Physics. , pp. 1429-1440, 1997.

[8] S. Sundet, "Morphology of the rejecting surface of aromatic polyamide membranes for desalination," Journal of Membrane Science, pp. 175-183, 1993.

[9] S.-Y. Kawk, S. Jung and S. Kim, "Structure-Motion-Performance Relationship of Flux-Enhanced Reverse Osmosis (RO) Membranes Composed of Aromatic Polyamide Thin Films," Environmental Science Technology , pp. 4334-4340, 2001.

[10] E. H. S. Vrijenhoek and M. Elimelech, "Influence of membrane surface properties on initial rate of colloidal fouling of reverse osmosis and nanofiltration membranes," Journal of Membrane Science, pp. 115-128, 2001.

[11] C. Tang, K.-N. Kown and J. Lockie, "Effect of membrane chemistry and coating layer on physicochemical properties of thin film composite polyamide RO and NF membranes I. FTIR and XPS characterization of polyamide and coating layer chemistry," Desalination, pp. 149-167, 2009.

[12] S.-Y. Kwak, "Relationship of relaxation property to reverse osmosis permeability in aromatic polyamide thin-film-composite membranes," Polymer, pp. 6361-6368, 1999.

[13] S. Kim, S.-Y. Kwak and T. Suzuki, "Positron Annihilation Spectoscopic Evidence to Demonstrate the Flux-Enhancement mechanism in Morphology-Controlled Thin-Film-Composite (TFC) Membrane," Envionmental Science Technology, pp. 1764-1770, 2005.

[14] O. Coronell and B. Z. X. C. D. Marinas, "Quantification of Functional Groups and Modeling of Their Ionization Behavior in the Active Layer of FT30 Reverse Osmosis Membrane," Environmental Science Technology, pp. 5260-5266, 2008.

[15] T. Sasaki, T. Mitsuhata, K. Takagi and K. Oto.USA Patent 20120248027 A1, 2012. 